\title{
Pregnant and non-pregnant women and low back pain-related differences on postural control measures during different balance tasks
}

\author{
Adriana Paula Fontana Carvalho1,2, Sébastien S. Dufresne², Márcio Rogério de Oliveira', Fernanda Kelly Sereniski Beraldo', \\ Pablo Eduardo Albuquerque de Souza', Rubia Stella da Silva', Maryane Dubois², Mathieu Dallaire², Suzy Ngomo², Rubens \\ Alexandre da Silva Jr1,2
}

\begin{abstract}
Introduction: Low back pain (LBP) is the most common musculoskeletal complaint in pregnancy, being responsible for many negative impacts. Objective: To evaluate the effect of LBP on static and dynamic balance in pregnant women and whether pregnancy mediates the results compared to non-pregnant women. Methods: 44 women (mean age $30 \mathrm{yrs}$ ) participated voluntarily in this study: 16 pregnant women with LBP starting in pregnancy, 14 pregnant women without LBP and 14 non-pregnant women as a group control. Participants were assessed for static postural balance using a force platform and dynamic mobility balance using the Timed Up and Go (TUG) test. Results: The pregnant women with LBP showed significant $(\mathrm{P}<0.04$, for mean, $d=1,2)$ poor postural balance in static tests (force platform), in the area of COP eyes open. In dynamic balance (TUG test), statistical difference was found between the groups (P 0.038 ) and the effect size were moderate to strong in the comparison between the three groups. The most sensitive differences were reported mainly between pregnant women with LBP versus non-pregnant control group in balance measures from force platform. Conclusion: The findings indicate that LBP associated to pregnant clinical status can decrease the balance capacity in women. These results have implication for balance evaluation and retraining in pregnant women with and without LBP from rehabilitation or prevention programs.
\end{abstract}

Keywords: Low Back Pain; Pregnancy; Postural Balance; Pain; Posture.

\section{BACKGROUND}

Low back pain (LBP) is one of the most frequent cause of disability affecting the worldwide adult population ${ }^{(1)}$. Clinical observations suggest that approximately $84 \%$ of adults will experience a LBP at some point in their life, in Brazil, each year, it was estimated that up to $65 \%$ of men and women in all ethnic groups suffered from LBP(2). LBP is defined as specific or non-specific axial or sagittal musculoskeletal pain located between L1 to L5 vertebrae with or without radiating leg symptom ${ }^{(3)}$. Pain severity correlates with function, those with more severe pain had poorer function while those with mild, well-controlled pain functioned normally ${ }^{(4)}$.

LBP genesis may have diverse origins, such as repetitive movements, postures, stress or exposition of prolonged static position, mechanical, hormonal, circulatory and psychosocial issues which are now well-known factors capable of increasing the risk of chronic pain ${ }^{(5)}$. Based on that fact, it is no surprise that the specific LBP estimated incidence differs during pregnancy. In fact, pregnancy-related LBP is a common symptom affecting $24 \%$ to $90 \%$ of these women worldwide. Because of the lack of knowledge and understanding of LBP, health care professionals do not know how to deal with or relieve patient's symptoms. LBP are often sufficient to alter normal physical and daily life activities ${ }^{(6,7)}$.

During the pregnancy period, many biological adaptations such as skeletal muscle mass, body dimensions and posture change the morphological characteristics of women and in turn, may increase spinal instability and generate back pain ${ }^{(8)}$. From a biomechanical perspective, these adaptations move the center of gravity (COG) to the anterior and superior poles in body and may contribute to balance disorders. In fact, static balance is the ability to keep the COG within the base of support and dynamic balance as the capacity to perform a task while restoring a stable COG position ${ }^{(9)}$. Under normal condition, balance performance is maintained by both static and dynamic controls. The pregnancy-related COG displacement can affect postural control strategies related to neuromuscular changes and changes in biomechanics for equilibrium of the body ${ }^{(10)}$. Apparently, postural alterations are evident during the gestational period, as well as expected changes in balance ${ }^{(10)}$. However, this phenomenon increases the risk of musculoskeletal discomfort in the thorax and lower limbs and consequently, causes foot, back and lower limb disorders, gait changes, poor 
mobility and pain ${ }^{(11)}$. Therefore, the repercussions of pregnancy on the musculoskeletal system result in great adjustments in static and dynamic posture for a woman ${ }^{(12)}$

Few studies have, however, compared balance and mobility in pregnant women with LBP and these results relative to a control non-pregnant group ${ }^{(11)}$. Opala-Berdzik et al. investigated static stability measures under different conditions in women between the onset of gestation, advanced gestation and 2 and 6 months post-gestation. These authors reported a significant negative balance effect in the advanced gestation group when compared to the non-gestational state ${ }^{(8)}$. It would be interesting to compare these results with pregnant women with LBP as well as with non-pregnant women.

The aim of this study was to compare static and dynamic balance in pregnant women with and without LBP and non-pregnant women. The hypothesis of this study was that pregnant women with LBP would present poorer functionality and postural balance than women without LBP or women not pregnant.

\section{METHODS}

\section{Design and participants}

This research was an exploratory and descriptive cross-sectional study with a convenience sample. The study included pregnant and non-pregnant women. For pregnant women with LBP, the inclusion criteria were: 1) women over 18 years old; 2 ) between 22 and 33 weeks of gestation; 3 ) being under prenatal clinical follow-up; 4) nonspecific LBP reported with Visual Analogic Scale (VAS) 3 to 10; 5) not having or participating in specific treatment (physical therapy or medication for LBP in the last 3 months); 6) able to perform functional activities, and not presenting limitations in relation to cognition and attention. For pregnant women without LBP, the inclusion criteria were: 1 ) women over 18 years old; 2 ) between 22 and 33 weeks of gestation; 3 ) being under prenatal clinical follow-up; 4) nonspecific LBP reported with VAS 0 to $2 ; 5$ ) not having or participating in specific treatment (physical therapy or medication for LBP in the last 3 months); 6) able to perform functional activities, and not presenting limitations in relation to cognition and attention. For non-pregnant women, were: 1) women over 18 years old; 2 ) not having gestation in the last 12 months; 3) without or not reporting LBP in the past 3 months; 4) able to perform functional activities and not presenting limitations in relation to cognition and attention. For both cases (pregnant and non-pregnant women) the exclusion criteria were: inability to perform the tests proposed or showing any condition that indicated a risk pregnancy. The study and measurement protocols were approved by the local ethics committee (number 1.579.189).

Based on a previous balance study with pregnant women using the center of pressure (COP) variable of the force platform: Velocity anteroposterior, pregnant women $=5.36 \pm 0.25 \mathrm{~mm} / \mathrm{s}$ and in non-pregnant women Velocity anteroposterior $=4.96 \pm 0.23 \mathrm{~mm} / \mathrm{s}$ ), the minimal sample size for a power of 0.95 at the 0.05 significance level was 11 participants per group ${ }^{(13)}$. A total of 30 pregnant and 14 non-pregnant women participated in this study. The pregnant women were divided into two groups according to VAS score for LBP, pregnant women with LBP (G1) for score between 3 and 10 ( $n=16$, age in mean 32; Standard Deviation: $S D=7 \mathrm{yrs})$, pregnant women without $L B P(G 2)$ for score between 0 and 2 ( $n=14$, age in mean 29; SD = 6 yrs), and non-pregnant women without LBP (G3), as a control group $(n=14$, age in mean $30 ; S D=7 \mathrm{yrs}$ ).

\section{Procedures and experimental protocol}

For the evaluation of participants, pregnant women, a standardized protocol was used for data collection including personal information, anthropometric measures, VAS, and obstetric history, as well as evaluation of the presence of pain through physical examination and tests of the mobility of the spine and pelvis ${ }^{(14)}$. For pregnant women were still used, three validated questionnaires were used to assess the clinical state: 1 ) disability related to pain using the Roland-Morris Disability Questionnaire (RMDQ) $)^{(15)}$ and the Oswestry Disability Index (ODI) ${ }^{(16)}$ and 2) the pain state using the McGill Pain Questionnaire (MPQ) ${ }^{(17)}$. To ensure a healthy control group (non-pregnant women), VAS, personal data and anthropometric measurements were collected. All participants in the control group reported zero on the VAS measure.

\section{Static balance assessment}

For static balance (Figure 1), we used postural control measures from a force platform (BIOMEC 400, EMG system do Brasil, SP, Ltda). Two static balance experimental task conditions were performed randomly: (1) two-legged stance with eyes

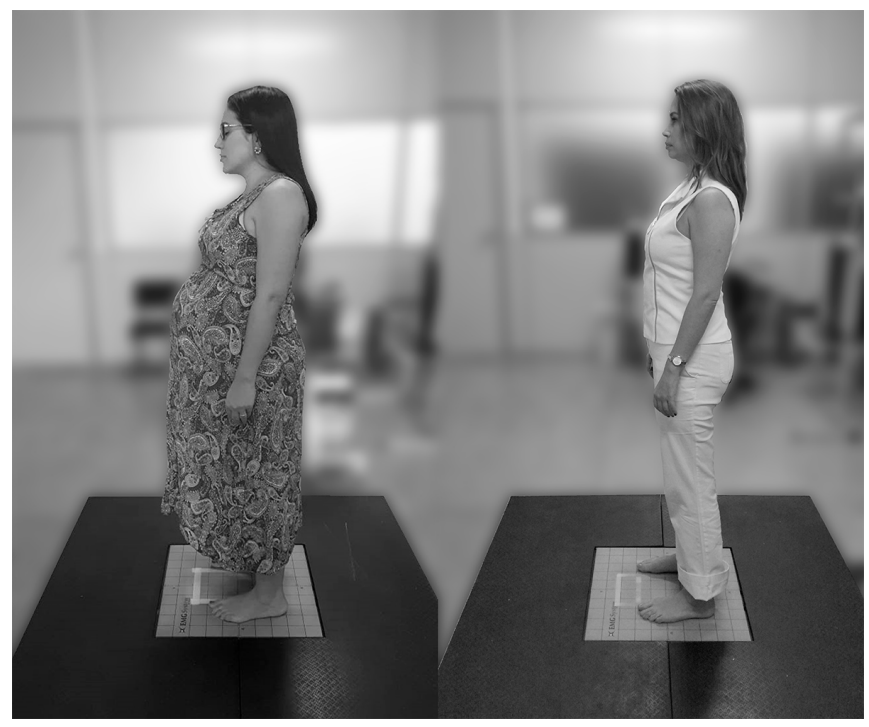

Figure 1. Illustration of a typical participant under force platform for balance measurement (BIOMEC 400). Note: Static balance experimental task conditions: two-legged stance with eyes open and with eyes closed. 50x42mm (300x300 DPI). 
open (TLEO); (2) two-legged stance with eyes closed (TLEC). All participants were familiarized with the equipment and balance protocol until they were comfortable with the testing. Balance assessment was performed with a standardized protocol: barefoot with arms at the sides or parallel to the trunk. During testing with eyes open, the participant would look at a target (a cross) placed on a wall at eye level $2 \mathrm{~m}$ away. To prevent falls during testing, an investigator stood close to the volunteers during all tasks. For each balance condition, three trials of $30 \mathrm{~s}$ with $30 \mathrm{~s}$ rest intervals were performed and the mean was retained for analysis ${ }^{(18)}$. A landmark on the force platform was used to standardize the position of the feet during all balance conditions.

The vertical ground reaction force data from the force platform was sampled at $100 \mathrm{~Hz}$. All force signals were filtered with a $35-\mathrm{Hz}$ low-pass second-order Butterworth filter. The signals from four sensors were converted into COP data using computerized stabilography, which was compiled with MATLAB routines (The Mathworks, Natick, MA). Stabilographic analysis of COP data led to the computation of the main balance parameters: the $95 \%$ confidence ellipse area of COP (A-COP in $\left.\mathrm{cm}^{2}\right)$, mean velocity (VEL in $\mathrm{cm} / \mathrm{s}$ ) in both anteroposterior $(\mathrm{A} / \mathrm{P})$ and mediolateral $(\mathrm{M} / \mathrm{L})$ directions of movement, and total displacement of $\operatorname{COP}(\mathrm{cm})^{(19)}$.

\section{Dynamic mobility balance assessment}

For physical mobility tests, we used the Timed Up and Go (TUG) test, which was undertaken using a chair with seat height of $46 \mathrm{~cm}$, back-support and armrests. The participants were oriented "to walk as fast as you can until you cross the cone, turn around, and walk back to the chair and sit down again". The cone was positioned $3 \mathrm{~m}$ from the patient's starting position. The time taken to complete the TUG was recorded in seconds using a chronometer bio Stopwatch (Model No. SW 2018). A total of two trials were assessed with 1 minute intervals between each trial, the best time performance being considered as a final measure ${ }^{(20)}$. A trained physiotherapist assessed the postural balance and TUG tests.

\section{STATISTICAL ANALYSES}

Categorical variables were described in absolute frequency (n) and relative frequency (\%), while numerical variables were described in mean and standard deviation (SD). Shapiro-Wilk test was used to evaluate the normality of the balance variables and determine which tests would be used. One-way ANOVA was used to assess differences between groups (pregnant women with LBP x pregnant women without LBP $x$ control) related to balance measures (force platform and TUG). The effect size ( $d$ ) between groups was calculated to determine the magnitude of effects using the equation: $d=\mathrm{m} 1-\mathrm{m} 2 / \mathrm{SDm} 2$, where $\mathrm{m} 1$ is the mean of the pregnant women with LBP group, while $\mathrm{m} 2$ is the mean of the control group, and $\mathrm{SDm}^{2}$ is the standard deviation of the control group. The effect size was characterized as by Cohen (2013) as weak, moderate and strong effects, i.e., $d=0.2$ is small, $d=0.5$ medium and $d=0.8$ large, respectively ${ }^{(21)}$. Spearman Correlation analysis was applied between the results of the questionnaires and the data of the force platform and TUG. All statistical analyses were performed with SPSS 20.0 for Windows (SPSS Inc., Chicago, IL, USA) with a level of significance of 0.05 .

\section{RESULTS}

The three groups were homogeneous for anthropometric characteristics (age, weight, height and body mass index), with no significant difference $(P>0.05)$ between them. Table 1 also shows the data related to parity and pain characteristics of women. The descriptive results (table 1 ) showed that $31 \%$ of G1 pregnant had a score that indicates disability by RMDQ. In the ODI questionnaires, $50 \%$ of G1 pregnant obtained scores 21 to 40 , indicating a moderate disability. The VAS pain score (0-10) for G1 pregnant had mean of 6, and for G2 pregnant was 1.5. In the questionnaire (MPQ) the most frequent type of pain for G1 pregnant was described "tiring / exhaustive" (76\%).

Pregnant women of G1 and G2 (pregnant) showed poor static and dynamic balance, for all variables analyzed when compared to $\mathrm{G} 3$ (no pregnant). The most significant and sensitive differences between groups were reported for the force platform measurement, in Area of COP eyes open, where $\mathrm{G} 1>\mathrm{G} 3 d=1,2$ and $\mathrm{G} 2>\mathrm{G} 3 d=0.90$ (P 0.040); while for the eyes closed, was no statistically significant difference, but the effect size was moderate to strong across these differences ( $\mathrm{G} 1>\mathrm{G} 3 d=0.83$ and $\mathrm{G} 2>\mathrm{G} 3 d=0.72$ ); in the other variables of the force platform there was no statistically significant difference between the groups (table 2) and the effect size was weak to moderate ( $G 1 \times G 3 d=0.7$ to $0.50 ; G 2 \times G 3 d=0.23$ to $0.53 ; \mathrm{G} 1 \times G 2 d=0.1$ to 0.27 ). For the TUG, statistical difference was found between the groups (P 0.038) and the effect size were moderate to strong in the comparison between the three groups (G1xG3 $d=0.86$; G2xG3 $d=0.53$; G1xG2 $d=0.54$ )

The results of correlations of clinical status (pain and disability scores) and COP parameters and TUG varied from weak to moderate across the groups, where among the questionnaires and variables of the platform $r$ was .36 to .43 , between questionnaires and TUG $r$ was .09 to .22 , and between platform and TUG $r$ was .00 to .26 .

\section{DISCUSSION}

This study aimed to compare the static and dynamic postural balance of pregnant women with and without LBP compared to non-pregnant women. LBP during pregnancy is one of the most common musculoskeletal condition. Similarly than epidemiological data, in the present investigation, 53\% of pregnant women experience significant LBP (VAS $\geq 3$ ). In addition, pregnant women with and without LBP have poor postural balance when compared to non-pregnant women, 
Table 1. Characterization of pregnant and non-pregnant women.

\begin{tabular}{|c|c|c|c|}
\hline \multirow{3}{*}{ Characteristic } & Pregnant & \multirow{2}{*}{$\begin{array}{c}\text { Pregnant } \\
\text { G2 }\end{array}$} & \multirow{2}{*}{$\begin{array}{c}\text { No pregnant woman } \\
\text { G3 }\end{array}$} \\
\hline & G1 & & \\
\hline & (16) & (14) & (14) \\
\hline Age (years) & $32(7)$ & $29(6)$ & $30(7)$ \\
\hline $\mathrm{BMI}\left(\mathrm{kg} / \mathrm{m}^{2}\right)$ & $25(2)$ & $25(3)$ & $24(5)$ \\
\hline VAS & $6(1)$ & $1.5(1)$ & 0 \\
\hline Gestational age (weeks) & $23(5)$ & $23(5)$ & - \\
\hline Uterine fundus $(\mathrm{cm})$ & $40(5)$ & $38(6)$ & - \\
\hline Abdominal circumference $(\mathrm{cm})$ & $91(11)$ & $93(8)$ & - \\
\hline Lumbar pain time (months) & $3(2)$ & $2(4)$ & - \\
\hline Primiparous & (5) $31 \%$ & (12) $85 \%$ & - \\
\hline Ocupation & & & - \\
\hline Housewife & (6) $37 \%$ & (2) $14 \%$ & - \\
\hline Teacher & (3) $19 \%$ & (4) $28 \%$ & - \\
\hline Student & (1) $6 \%$ & (2) $20 \%$ & - \\
\hline Other ocupation & (6) $37 \%$ & (6) $43 \%$ & - \\
\hline Radiated pain reported & (7) $48 \%$ & (2) $14 \%$ & - \\
\hline Activity practice before pregnancy & (7) $48 \%$ & (7) $50 \%$ & - \\
\hline Have pain in rest & (13) $81 \%$ & (4) $29 \%$ & - \\
\hline Have night pain & (5) $31 \%$ & (3) $21 \%$ & - \\
\hline \multicolumn{4}{|l|}{ What makes the pain worst? } \\
\hline Seated position & (2) $12 \%$ & (4) $29 \%$ & - \\
\hline Standing position & (2) $12 \%$ & (2) $14 \%$ & - \\
\hline Maintain position for an extended period & (4) $25 \%$ & (1) $7 \%$ & - \\
\hline Others & (8) $50 \%$ & (1) $7 \%$ & - \\
\hline \multicolumn{4}{|l|}{ What makes the pain better? } \\
\hline Rest & (7) $48 \%$ & (6) $43 \%$ & - \\
\hline Change position & (3) $19 \%$ & (1) $7 \%$ & - \\
\hline Others & (6) $37 \%$ & (1) $7 \%$ & - \\
\hline \multicolumn{4}{|l|}{ Roland Morris Questionnaire } \\
\hline From 0 to 6 points & (3) $19 \%$ & (7) $50 \%$ & - \\
\hline From 7 to 13 points & (8) $50 \%$ & (4) $29 \%$ & - \\
\hline From 14 or more points & (5) $31 \%$ & (3) $21 \%$ & - \\
\hline \multicolumn{4}{|l|}{ Oswestry Questionnaire } \\
\hline From 0 to $20 \%$ & (5) $31 \%$ & (9) $64 \%$ & - \\
\hline From 21 to $40 \%$ & (8) $50 \%$ & (4) $29 \%$ & - \\
\hline From 41 to $60 \%$ & (3) $19 \%$ & (2) $14 \%$ & - \\
\hline
\end{tabular}

Note: Low Back Pain (LBP); Body Mass Index (BIM); Visual Analogue Scale (VAS). Mean (Standard deviation) of the characterization pregnant women with $\operatorname{LBP}(\mathrm{G} 1)$, without LBP (G2) and no pregnant and without pain $(\mathrm{G} 3)$, in relation to anthropometric and personal data, percentage of characterization of pain, and pain and disability scores.

and the results for the effect size for these differences, indicating that LBP is a factor for decreasing balance in pregnant women. To the authors' knowledge, there are few studies that to compare three different groups of women in the same experimental design, which supports the originality of the results found. Past studies addressed this question separately, analyzing different gestational periods ${ }^{(5)}$ or LBP effects post-childbirth ${ }^{(22,23)}$

In brief, the characteristics of our sample (Table 1) were similar to the other studies related to LBP in pregnancy ${ }^{(5,6,24)}$, as well as the factors that interfere in the improvement or worsening of pain ${ }^{(25)}$. The LBP women group had a moderate 
Tabela 2. Mean (Standard deviation) for timed up go test and postural balance variables.

\begin{tabular}{|c|c|c|c|c|c|c|}
\hline \multicolumn{2}{|c|}{ Postural balance } & \multirow{2}{*}{$\begin{array}{c}\text { Pregnant } \\
\text { G1 }\end{array}$} & \multirow{2}{*}{$\begin{array}{c}\text { Pregnant } \\
\text { G2 }\end{array}$} & \multirow{2}{*}{$\begin{array}{c}\text { No pregant } \\
\text { G3 }\end{array}$} & \multirow{2}{*}{$\frac{\text { Anova }}{\mathrm{F} \text { (P value })}$} & \multirow{2}{*}{$\begin{array}{c}\text { Tukey } \\
\text { (direction) }\end{array}$} \\
\hline & & & & & & \\
\hline \multirow{2}{*}{ A-COP $\left(\mathrm{cm}^{2}\right)$} & EO & $2.34(1.54)$ & $2.94(2.80)$ & $1.11(0.64)$ & $3.48(0.04)^{*}$ & $<0.05$ (Pregnant G1 $>$ Control) \\
\hline & EC & $4.28(5.1)$ & $2.87(3.34)$ & $1.15(0.55)$ & $2.78(0.73)$ & $<0.05$ (Pregnant G1 $>$ Control) \\
\hline \multirow{2}{*}{$\operatorname{VEL~A/P~}(\mathrm{cm} / \mathrm{s})$} & EO & $0.73(0.21)$ & $0.76(0.20)$ & $0.71(0.13)$ & $0.32(0.72)$ & -- \\
\hline & EC & $0.92(0.21)$ & $0.93(0.25)$ & $0.83(0.16)$ & $1.01(0.37)$ & -- \\
\hline \multirow{2}{*}{ VEL M/L (cm/s) } & EO & $0.55(0.07)$ & $0.53(0.08)$ & $0.51(0.09)$ & $0.72(0.49)$ & -- \\
\hline & EC & $0.59(0.07)$ & $0.63(0.29)$ & $0.53(0.17)$ & $1.13(0.33)$ & -- \\
\hline \multirow{2}{*}{ Total-D (cm) } & EO & $61.84(10.85)$ & $61.2(13.54)$ & $57.23(9.18)$ & $0,70(0.50)$ & -- \\
\hline & EC & 72.03 (11.98) & $71.59(15.54)$ & $64.69(10.11)$ & $1.50(0.23)$ & -- \\
\hline TUG (s) & & $7.51(1.93)$ & $6.67(0.92)$ & $6.20(0.85)$ & $3.55(0.03)^{*}$ & $<0.05$ (Pregnant $\mathrm{G} 1>$ Control) \\
\hline
\end{tabular}

Note: Timed up and go test (TUG); Area of center of pressure (A-COP); COP velocity in the anteroposterior (VEL A/P) and mediolateral (VEL M/L) directions; Total displacement (Total-D); Low back pain (LBP); Eyes open (EO); Eyes closed (EC). *Significantly different.

disability and pain intensity level (Table 1). However, correlations between clinical status (disability and pain questionnaires) and measures of balance were poor to moderate. These results confirm that changes in balance measures may be related to a combined effect of pregnancy itself and the pathology of LBP. Moreira et al, evaluated 15 pregnant women with LBP and 15 non-pregnant women, concluded that the changes in the postural oscillation of the pregnant woman may be related to changes in biomechanical and hormonal levels, in addition, he further concluded that postural control during static posture can't be used to predict the occurrence of LBP(13).

Our results for balance are in agreement with some studies ${ }^{(9,18,19)}$ and in disagreement with others ${ }^{(10,12,23)}$. For example, Opala-Berdzik et al evaluated 31 pregnant women without LBP and reported no effect on postural balance with eyes open. These authors reported an effect of an increase of postural instability only with the eyes closed and during advanced pregnancy (around the 36th week), which is contrary to the results of the present study, which found a decrease in both open and closed eyes, and also found that this balance decreases as LBP is higher ${ }^{(24)}$.

We observed to a statistical difference in the TUG test when comparing pregnant women with LBP and non-pregnant women (Table 2), our results showed that pregnancy itself worsens mobility, but the presence of LBP has impact on the decrease of mobility during pregnancy. This is in agreement with a previous study which showed that difficulties in sitting and standing are significant in pregnancy and that these conditions may worsen due to LBP(6). All together, these results suggest that effects on balance can be task dependent. More challenging balance performance and sophisticated equipment are suitable to better discriminate differences between pregnant women with LBP and non-pregnant women, as the present study did. In this sense, the strength of our experimental protocol was the use of a clinical protocol related to direct postural control measurements through a stabilographic analysis on a force platform, which determined the difference in balance across groups.

On the other hand, the causes of LBP are not fully understood, but during pregnancy, we must consider a series of factors related to hormonal and biomechanical changes ${ }^{(25)}$, as well as other factors related to increase in body mass index, decreased muscle strength and center of gravity changes ${ }^{(22,23)}$. According to Vermani et al., an increase of movement from pelvic joints in pregnant women, decreases the efficiency of load transmission, increases shearing forces between pelvic joints and leads to a higher probability of pain ${ }^{(26)}$. In fact, one explanation for poor balance in pregnant women with LBP can be associated with these factors.

In addition, Panjabi's model(27) can also explain at least some of our results. Some studies of people with LBP have reported significantly poorer balance across this population when compared with health controls ${ }^{(19)}$. The differences between the groups with and without LBP may be related to trunk muscle fatigue in individuals with LBP, resulting in brief uncontrolled intervertebral movements and postural instability ${ }^{(28)}$. Individuals with LBP present low levels of trunk muscle resistance compared to controls ${ }^{(29)}$ In addition, impaired lumbosacral proprioception is also associated with LBP ${ }^{(30)}$, which in turn affects balance performance.

We found a large effect size for the differences between groups of pregnant women with LBP and without LBP, compared to control, but we should note that the effect size was higher when we analyzed the group with LBP compared to the control, which indicates that pregnancy itself is a sufficient factor for decreasing the balance, and the presence of LBP increases this instability. However, currently there is no normative data for what is the minimal clinically important difference (MCID) in balance based on center of pressure measures. This is an important topic and future studies are needed to address this scientific gap. 
Finally, the clinical implications of our results is a greater understanding of LBP characteristics during pregnancy and its repercussions, and thus can guide the professional in the formulation of physiotherapeutic objectives and activities that address the set of symptoms and deficits that pregnant women with LBP experience. Another implication is to demonstrate that pregnancy associated with LBP is a determinant factor in the decrease in functionality and the presence of an incapability level as shown in the results from the applied questionnaires. Thus, physiotherapists could better address balance evaluation and retraining for pregnant women with LBP in prevention or rehabilitation programs.

One limitation of this study was to find pregnant women totally without LBP. After a more careful evaluation, even with the absence of self-reported pain, the anamnesis and the physical examination detected the presence of a minimal level, so the study accepted the level of VAS $\leq 2$, as absence of pain. Other equilibrium tests (eg, semi-tandem) were not evaluated. Therefore, the results may not be generalizable for the entire population. However, we combined participants in significant demographic variables to reduce potential confounding factors, but other variables not used in the match may still have affected comparability between groups.

\section{CONCLUSION}

Pregnant women with and without LBP presented poor postural control and lower mobility when compared to non-pregnant women, where the presence of LBP increased these differences. These results have implications for the assessment of balance and retraining in pregnant women with and without LBP from rehabilitation or prevention programs.

\section{AUTHOR'S CONTRIBUTIONS}

APFC conceived of the study, and participated in its design and coordination, and drafted the manuscript; MRO participated in the design of the study and performed the statistical analysis, and helped to draft the manuscript; FKSB, PEAS and RSS participated in the collection of evaluation data and experimental protocol; SSD, MD, MD and SN participated in its design and helped to draft the manuscript; RAS conceived of the study, and participated in its design and coordination, performed the statistical analysis and drafted the manuscript. All authors read and approved the final manuscript.

\section{CONFLICT OF INTEREST}

The authors declare that they have no competing interests.

\section{AUTHORS DETAILS}

1. Universidade Pitagoras (UNOPAR), Londrina (PR), Brasil.

\section{REFERENCES}

1. Leboeuf-Y C, Nielsen J, Kyvik KO, Fejer R, Hartvigsen J. Pain in the lumbar, thoracic or cervical regions: Do age and gender matter? A population-based study of 34,902 danish twins $20-71$ years of age. BMC Musculoskelet Disord. 2009;10(1):39.
2. De Jesus-Moraleida FR, Ferreira PH, Ferreira ML, Da Silva JP, Assis MG, Pereira LSM. The brazilian back complaints in the elders (brazilian BACE) study: Characteristics of brazilian older adults with a new episode of low back pain. Braz J Phys Ther. 2017; 22(1):55-63.

3. Allegri M, Montella S, Salici F, Valente A, Marchesini M, Compagnone $C$, et al. Mechanisms of low back pain: a guide for diagnosis and therapy. F1000Res, v. 5, 2016.

4. Silver JK, Baima J, Mayer RS. Impairment-driven cancer rehabilitation: an essential component of quality care and survivorship. CA Cancer J Clin. 2013;63(5):295-317.

5. Paula LFd, Silva RGC, Andres LF, Korelo RIG. Association between kinesiologic dysfunctions, lumbar disability and lumbopelvic pain in pregnancy. Fisioter Mov. 2017;30(3):473-484.

6. Glinkowski WM, Tomasik P, Walesiak K, Głuszak M, Krawczak K, Michoński $\mathrm{J}$, et al. Posture and low back pain during pregnancy-3D study. Ginekol Pol. 2016;87(8):575-580

7. Pennick V, Liddle SD. Interventions for preventing and treating pelvic and back pain in pregnancy. Cochrane Database Syst Rev. 2013;8.

8. Opala-Berdzik A, Bacik B, Cieślińska-Świder J, Plewa M, Gajewska M. The influence of pregnancy on the location of the center of gravity in standing position. J Hum Kinet.2010;26:5-11.

9. Pollock AS, Durward BR, Rowe PJ, Paul JP. What is balance? Clin Rehabil. 2000;14(4):402-

10. Takeda K, Shimizu K, Imura M. Changes in balance strategy in the third trimester. J Phys Ther Sci. 2015;27(6):1813-1817.

11. Ritchie JR. Orthopedic considerations during pregnancy. Clin Obstet Gynecol. 2003;46(2):456-466.

12. Ribas $S$, Guirro E. Analysis of plantar pressure and postural balance during different phases of pregnancy. Braz J Phys Ther. 2007;11(5):391-396.

13. Moreira LS, Elias LA, Gomide AB, Vieira MF, Do Amaral WN. A longitudinal assessment of myoelectric activity, postural sway, and low-back pain during pregnancy. Acta Bioeng Biomech. 2017;19(3):77-83.

14. Vleeming A, Albert HB, Östgaard HC, Sturesson B, Stuge B. European guidelines for the diagnosis and treatment of pelvic girdle pain. Eur Spine J. 2008;17(6):794-819.

15. Nusbaum L, Natour J, Ferraz MB, Goldenberg J. Translation, adaptation and validation of the roland-morris questionnaire-brazil roland-morris. Brazilian Braz. J. Med. Biol. Res. 2001;34(2):203-210.

16. Falavigna A, Teles AR, de Braga GL, Barazzetti DO, Lazzaretti L, Tregnago AC. Instrumentos de avaliação clínica e funcional em cirurgia da coluna vertebral instrumentos de evaluación clínica y funcional en cirugía de la columna vertebral instruments of clinical and functional evaluation in spine surgery. Coluna/Columna. 2011;10(1):62-67.

17. Costa LDCM, Maher CG, McAuley JH, Hancock MJ, de Melo Oliveira W, Azevedo DC, et al. The brazilian-portuguese versions of the McGill pain questionnaire were reproducible, valid, and responsive in patients with musculoskeletal pain. J Clin Epidemiol. 2011;64(8):903-912.

18. Da Silva RA, Bilodeau M, Parreira RB, Teixeira DC, Amorim CF. Age-related differences in time-limit performance and force platform-based balance measures during one-leg stance. J Electromyogr Kinesiol. 2013;23(3):634639.

19. da Silva RA, Vieira ER, Fernandes KB, Andraus RA, Oliveira MR, Sturion $\mathrm{LA}$, et al. People with chronic low back pain have poorer balance than controls in challenging tasks. Disabil Rehabil. 2018: 40(11):1294-1300.

20. Evensen NM, Kvåle A, Brækken IH. Reliability of the timed up and go test and Ten-Metre timed walk test in pregnant women with pelvic girdle pain. Physiother Res Int. 2015;20(3):158-165.

21. Cohen J. Statistical power analysis for the behavioral sciences. Routledge, 2013.

22. Gomes GRA, Araújo RCD, Lima AS, Pitangui ACR. Gestational low back pain: Prevalence and clinical presentations in a group of pregnant women. Rev dor. 2013;14(2):114-117. 
23. Madeira HGR, Garcia JBS, Lima MVV, Serra HO. Incapacidade e fatores associados à lombalgia durante a gravidez. Rev Bras Ginecol Obstet. 2013;35(12):541-548.

24. Opala-Berdzik A, Błaszczyk JW, Bacik B, Cieślińska-Świder J, Świder D, Sobota $G$, et al. Static postural stability in women during and after pregnancy: A prospective longitudinal study. PLoS One. 2015;10(6):e0124207.

25. Schröder G, Kundt G, Otte M, Wendig D, Schober H. Impact of pregnancy on back pain and body posture in women. J Phys Ther Sci. 2016;28(4):1199-1207.

26. Vermani E, Mittal R, Weeks A. Pelvic girdle pain and low back pain in pregnancy: a reviewPain Pract. 2010;10(1):60-71.
27. Panjabi MM. The stabilizing system of the spine. Part I. Function, dysfunction, adaptation, and enhancement. J Spinal Disord. 1992;5:383-383.

28. Parreira RB, Amorim CF, Gil AW, Teixeira DC, Bilodeau M, Da Silva RA. Effect of trunk extensor fatigue on the postural balance of elderly and young adults during unipodal task. Eur J Appl Physiol. 2013;113:1989-1996.

29. da Silva RA., Vieira ER, Cabrera M, Altimari LR, Aguiar AF, Nowotny $\mathrm{AH}$, et al. Back muscle fatigue of younger and older adults with and without chronic low back pain using two protocols: a case-control study. J Electromyogr Kinesiol. 2015;25:928-936.

30. Brumagne S, Janssens L, Knapen S, Claeys K, Suuden-Johanson E. Persons with recurrent low back pain exhibit a rigid postural control strategy. Eur Spine J. 2008;17:1177-1184. 